\title{
Treating Comorbid Insomnia in Older Adults via Cognitive-Behavioural Treatment, Bright Light and Exercise
}

\author{
K. Richter ${ }^{1,2 *}$, J. Myllymaeki' ${ }^{1}$, S. Scharold-Schaefer ${ }^{3}$, I. Tomova ${ }^{1}$, R. Mayrer ${ }^{4}$, \\ G. Niklewski ${ }^{1}$ \\ ${ }^{1}$ Clinic for Psychiatry and Psychotherapy, Nuremberg Hospital, Nuremberg, Germany \\ ${ }^{2}$ Faculty for Social Sciences, Georg-Simon-Ohm University for Applied Sciences, Nuremberg, Germany \\ ${ }^{3}$ Psychotherapeutical Practice, Weissenburg, Germany \\ ${ }^{4}$ Sozialstiftung Bamberg, Clinic for Psychiatry, Psychosomatic and Psychotherapy, Clinic Bamberg, Bamberg, \\ Germany \\ Email: ${ }^{*}$ Kneginja.Richter@gmx.de
}

Received 25 February 2014; revised 29 March 2014; accepted 7 April 2014

Copyright (C) 2014 by authors and Scientific Research Publishing Inc.

This work is licensed under the Creative Commons Attribution International License (CC BY).

http://creativecommons.org/licenses/by/4.0/

(c) (i) Open Access

\begin{abstract}
Background: Insomnia occurs frequently in older adults. The underlying factors for the disorder are physiological changes of the sleep-wake cycle, comorbid diseases and drug treatment. Cognitive behavioural therapy (CBT I) combined with light therapy and physical activity can be effective in the treatment of comorbid insomnia in older adults. Methods: Sixty-three (63) insomnia patients (47 female, 16 male) with an average age of 66.6 years participated in the program. Before and after the treatment, the participants completed questionnaires to assess their sleep quality (PSQI), day-time sleepiness (ESS), mood (BDI) and well-being (WHO-5-Index). In addition, they kept sleep diaries for six weeks. Results: Pre-post comparisons revealed a significant improvement in sleep latency and sleep quality, as well as a significant reduction of day-time sleepiness. In patients with both insomnia and depression, all depression scores improved slightly but significantly. Conclusions: Non-pharmacological combination treatment using CBT I, light therapy and physical activity seemed to be effective in older adults with comorbid insomnia and improve additionaly depressive symptoms.
\end{abstract}

\section{Keywords}

Insomnia, Non-Pharmacological Treatment, Older Adults, CBT, Light Therapy, Physical Activity

Corresponding author. 


\section{Introduction}

The goal of this study is to proof the efficiency of the non-pharmacological combination treatment including CBT, light therapy and physical activity for older adults aged 58 and above who suffer from chronic comorbid insomnia.

Almost half of all people aged over 65 are suffering from insomnia [1]-[7]. Their main complaints are difficulties with falling and/or remaining asleep and increased day-time sleepiness [8]. This insomnia can be caused by several age-related changes [9]-[11]. The reasons for the high prevalence of insomnia among older adults include physical and mental comorbid diseases and the use of medications [11]-[13]. It is difficult to assess retrospectively whether psychophysiological insomnia or physical illness occurs first. The data from the American EPESE longitudinal study with a three-year follow-up (National Institute on Ageing's established Population of Epidemiologic Studies of the Elderly) confirmed that $57 \%$ of elderly people suffer from at least one chronic sleep disorder, and the data indicate a close connection between insomnia and the occurrence of depressive mood, breathing disturbances, poor health and restricted physical capability [10] [14].

Another reason for aging-related changes in the sleep profile may be traced back to changes in sleep-wake regulation [15]. In both younger and older insomnia patients, melatonin levels are lower than in healthy people. With the increasing age, the amount of released melatonin decreases [16] [17], which causes changes in the sleep-wake cycle that appears as forward displacements of the sleep phase and a reduction in the amplitude of day-night oscillations. In addition, insufficient bodily activity in older people also contributes to the occurrence of insomnia symptoms. Figure 1 shows a summary of the reasons for the increased occurrence of sleep disorders in older adults.

According to prospective studies [6] [7], insomnia has a significant association with depression. Between $70 \%$ and $90 \%$ of patients with medium to severe depression suffer from severe sleep disorders. These mood disorders also correlate with anxiety symptoms and an increased risk for suicidal behaviour [18].

Increasing severity of sleep disorders is associated with an increased risk of a subsequent depressive condition [19].

Cognitive-behavioural therapy appears to be the most effective non-pharmacological therapy of insomnia. It includes several behavioural-therapeutic techniques such as sleep hygiene, stimulus control with bed-time restriction and cognitive restructuring [20]-[23]. There is good evidence for a positive therapeutic effect of each component of the behavioural-therapeutic measures such as stimulus control or sleep restriction, as well as for combination treatment consisting of cognitive-behavioural therapy with different muscular relaxation techniques $[24]$.

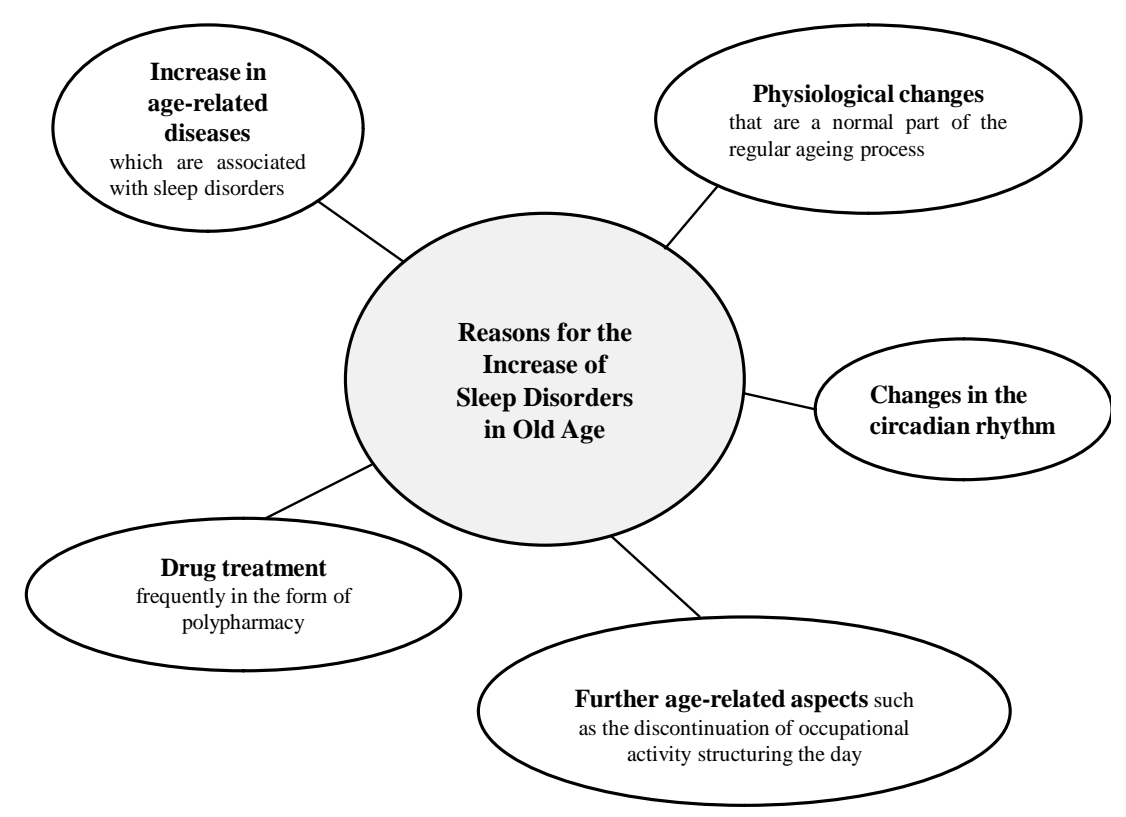

Figure 1. Reasons for the increase of sleep disorders in old age. 
Moreover, the effectiveness of each single technique has been examined as well: muscle relaxation technique, imaginery technique, sleep hygiene, sleep education, physical exercise, physical activity, massage and light therapy. The evidence-based criteria for good efficiency exist mostly for sleep restriction and sleep compression as single techniques [2].

Similarly, the best evidence for the non-pharmacological insomnia treatment in older adults exists for sleep restriction/sleep compression and stimulus control [25]-[30].

In particularly, the method of stimulus control appears to be effective which results in both the remission of insomnia in older adults and the long-term effects of up to two years [31].

Other single techniques as muscle relaxation therapy, meditation, bio-feedback, diaphragm breathing and visualisation have been widely used as a part of cognitive-behavioral treatment of insomnia with uncertain effect [2] [28].

Physical exercise and sports have shown a positive effect on the time to fall asleep and the quality of sleep in older adults, but there are no significant improvements in sleep efficiency. Moreover, the physical activity contributes to an improved quality of life through better sleep and other factors [32]-[34].

Light therapy has been also used as supportive method in insomnia treatment and requires that the patient spends approximately two hours each day in front of a light unit with an exposure to light of up to 10,000 lux. The timing of the light exposition should be aligned to the sleep-wake rhythm of the patient.

The effect of heliotherapy is supported by studies, which essentially consist of the application of light in the evening between 7 and 9 o'clock [35]-[37].

The non-pharmacological measures used have been demonstrated not only to improve the symptoms of primary insomnia, but also to alleviate and even prevent depressive symptoms in patients suffering from comorbid insomnia [19] [38]-[41].

\section{Methods}

Assessment was based on the data of 63 patients (47 female, 16 male) aged between 58 and 90 years (average age 66.6 years) diagnosed with primary or secondary non-organic insomnia (F51.0) who participated in group sleep treatment for older adults. The 63 patients were recruited from seven treatment groups over a period of four years (2005-2009) at the Center for Sleep Medicine, Nuremberg Hospital. The group sleep training sessions were offered twice a year (spring and autumn). The groups consisted of seven to ten patients. Informed consent was obtained from the participators.

The group sleep training covered a period of 10 weeks with six group meetings taking place at bi-weekly intervals. Before starting the group therapy, the patients had a consultation with a psychiatrist. The group meetings were chaired by psychiatrist and psychologist and had a duration of 90 minutes. In these meetings, participants were informed about the sleeping habits conducive to sleep ("sleep hygiene" and "stimulus control", modified sleep restriction) and rituals to aid in falling asleep. Moreover, they learned methods for physical and mental relaxation (progressive muscle relaxation using the Jacobson method as well as meditation [42].

After the fifth group meeting, a five-day "activation week" followed, during which participants met on a daily basis for light therapy in the morning (group setting, 45 minutes by 10,000 lux) followed by a joint activity (dance therapy, Nordic Walking, exercise, excursions and museum visits) (Table 1). Both the light therapy and the activating physical activities were performed to re-stabilise the sleep-wake rhythm of the elderly patients.

All patients were asked not to alter their medication during the training unless in the case of an urgent medical indication. Likewise, patients were asked not to initiate psychotherapy during the course of the sleep training.

The statistical analyses is based on the instruments listed below. The included case numbers differ depending on the existing database (between 39 and 47 cases). As a success control, a psychometric examination was conducted both before and after the training covering the parameters of sleep quality (Pittsburgh Sleep Quality Index, PSQI) [43], day-time sleepiness (Epworth Sleepiness Scale, ESS) [44], depressive symptoms (Beck Depressions Inventory, BDI or BDI II) [45] [46] and general well-being (questionnaire on general well-being, WHO-5) [47]. The BDI was administered to the patients from the first five groups. The other two groups received the revised version BDI II. Moreover, participants started keeping a sleep diary two weeks before the beginning of the group sleep training and kept it throughout the entire therapy period. Pre-post-comparisons were conducted with regard to the sleep protocols as well as total scores and sub scores of the questionnaires used (t-test for dependent samples). Because of the low number of samples for the comparison of the mean values of the BDI II, a Wilcoxon test was performed. We used SPSS 14.0 for the calculation of the data. 
Table 1. Schedule of the individual sessions.

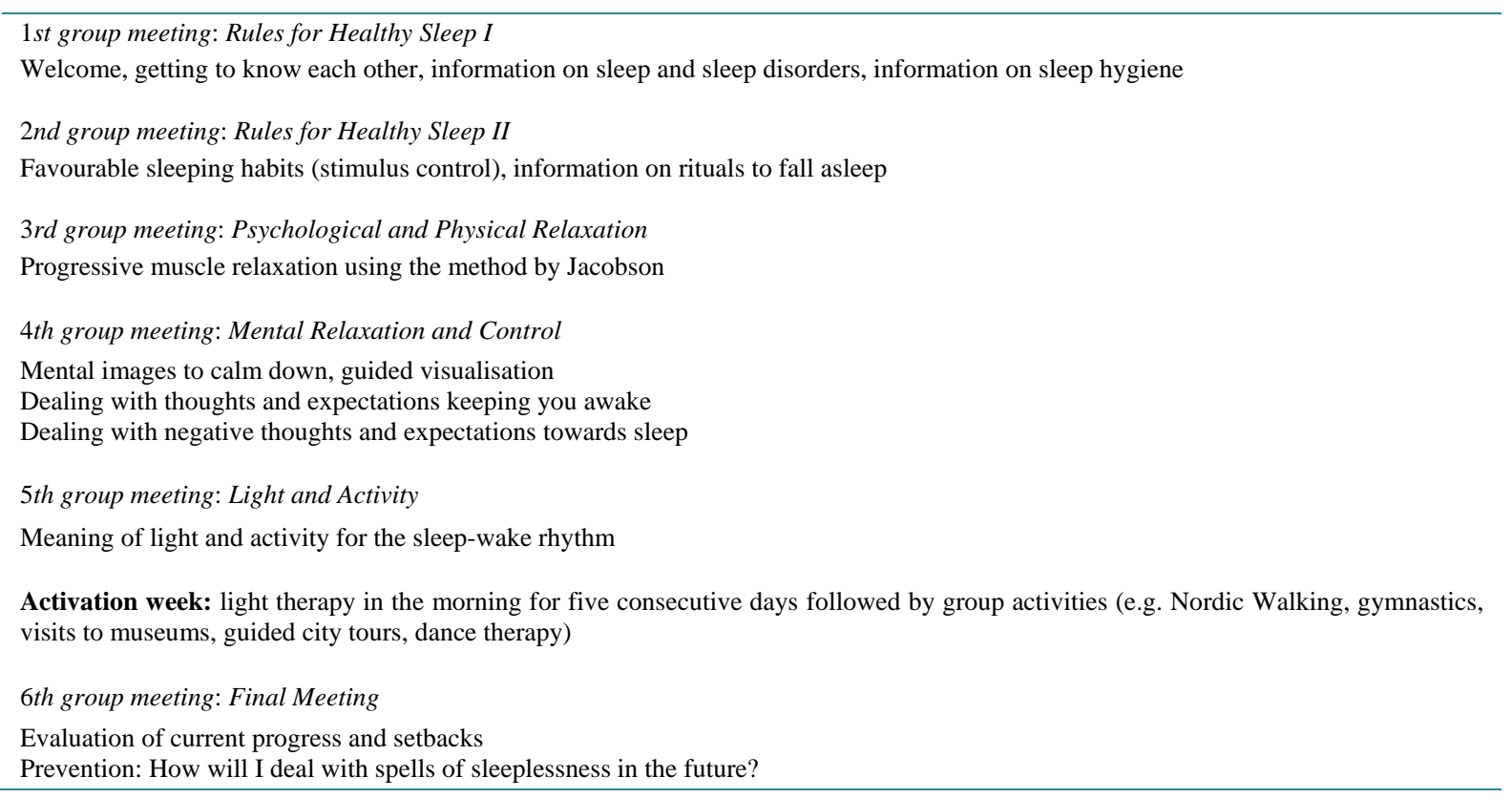

\section{Results}

The pre-post-comparison of the mean values of the ESS, the PSQI and the subscores on sleep quality and sleep latency revealed significant improvements after the cognitive-behavioural treatment in the group setting (Alpha error level after Bonferroni correction was 0.0028) (Table 2, Figure 2). The BDI score improved and almost reached the non-pathological score level for 34 patients, and the BDI II score reached the non-pathological score for 14 patients (Table 2, Figure 2). The patient Who-5 scores also improved to non-pathological score levels.

The pre-post-comparison of the mean values of the central components of the sleep protocols (day sleep, sleep latency, frequency of waking up, time awake, total time in bed, total sleep time, sleep efficiency) displayed no significant differences after Bonferroni correction (Table 3).

The comorbid psychiatric diagnoses based on ICD-10 are follows: 26 cases of depressive episode (ICD-10: F32), 20 cases of recurrent depressive disorder (ICD-10: F33). In addition, there was one case each of dysthymia (ICD-10: F34.1) and bipolar disorder (ICD-10: F31). The most common comorbid diagnoses among physical illnesses were sleep apnoea syndrome $(\mathrm{N}=17)$ and hypertension $(\mathrm{N}=20)$ (Table 4). Tricyclic antidepressants $(\mathrm{N}=19)$ and atypical benzodiazepines $(\mathrm{N}=16)$ were the most taken drugs (Table 4).

\section{Discussion}

Older Adults frequently suffer from difficulties with falling and/or remaining asleep [1] [7]. These issues are often connected with a lack of well-being, increased day-time sleepiness and cognitive disorders. Sleep disorders are related to increased morbidity and mortality. Therefore, healthy sleep is an essential precondition for maintaining the physical and mental performance and health of elderly people.

The ageing process does not cause sleep disorders, but it may be considered a risk factor for the occurrence of sleep disorders.

Long-term pharmacological therapy with hypnotics is not advisable because of the habituation effect and the risk of addiction [25]. Data prove that drug treatment is the most frequent form of therapy for sleep disorders in older age despite the concomitant problems. Before drug treatment is initiated, however, the possibilities of treating insomnia by non-pharmacological treatments should be considered and administered before or in combination with drug treatment. Treatment of sleep disorders will not be successful unless combined with sleephygienic and further non-pharmacological measures [7] [11].

Light therapy and behavioural-therapeutic measures are important elements of a multimodal treatment setting for insomnia, whereas sleep hygiene should include regular social and physical activity [37]. 
Table 2. Results of the scores of the testing procedures and the pre-post-comparisons.

\begin{tabular}{|c|c|c|c|}
\hline & PRE: M (SD) & POST: M (SD) & $\mathbf{p}$ \\
\hline ESS $(n=47)$ & $7.4(5.1)$ & $5.8(4.7)$ & $0.000^{*}$ \\
\hline PSQI total score $(n=46)$ & $13.1(3.1)$ & $11.1(3.4)$ & $0.000^{*}$ \\
\hline PSQI sleep quality $(n=47)$ & $1.9(0.5)$ & $1.5(0.5)$ & $0.000^{*}$ \\
\hline PSQI sleep latency $(n=46)$ & $2.4(0.9)$ & $1.8(1.0)$ & $0.000^{*}$ \\
\hline PSQI sleep duration $(n=46)$ & $5.1(1.3)$ & $5.3(1.0)$ & 0.145 \\
\hline PSQI sleep efficiency $(n=47)$ & $2.4(0.9)$ & $2.0(0.9)$ & 0.004 \\
\hline PSQI sleeping Disorders $(n=46)$ & $1.6(0.6)$ & $1.4(0.7)$ & 0.048 \\
\hline PSQI mean sleep consumption $(n=47)$ & $1.6(1.3)$ & $1.2(1.3)$ & 0.022 \\
\hline PSQI day-time tiredness $(n=46)$ & $1.3(0.7)$ & $1.1(0.7)$ & 0.128 \\
\hline WHO-5 $(n=41)$ & $12.3(4.8)$ & $14.0(4.5)$ & 0.020 \\
\hline BDI $(n=34$, groups 1 - 5) & 14.9 (7.9) & $11.2(7.2)$ & $0.001^{*}$ \\
\hline BDI II (n = 14, groups 6 and 7) & $12.3(7.9)$ & $10.4(9.3)$ & 0.274 \\
\hline
\end{tabular}

Table 3. Results of central components of the sleep protocols.

\begin{tabular}{|c|c|c|c|}
\hline & PRE & POST & $\mathbf{p}$ \\
\hline Daytime sleep $(\min ).(n=43)$ & $8.6(14.4)$ & $4.6(10.0)$ & 0.028 \\
\hline Sleep latency $(\min ).(n=45)$ & $42.7(28.7)$ & $33.7(26.0)$ & 0.023 \\
\hline Frequency of waking up $(n=44)$ & $2.4(1.4)$ & $2.3(1.6)$ & 0.582 \\
\hline Waking hours (min.) $(n=40)$ & $52.1(40.0)$ & 48.5 (39.7) & 0.639 \\
\hline Total time spent in bed $(h)(n=39)$ & $8.2(0.83)$ & $8.0(0.85)$ & 0.118 \\
\hline Total sleep time $(h)(n=39)$ & $5.3(1.1)$ & $5.5(1.1)$ & 0.117 \\
\hline Sleep efficiency (\%) (n = 39) & $64.6(13.2)$ & $68.4(13.8)$ & 0.047 \\
\hline
\end{tabular}

Table 4. Frequency and percentage data of the comorbid disorders and medication at the beginning of group training (based on the total sample $\mathrm{N}=63$ ).

\begin{tabular}{cccc}
\hline Comorbid diseases & $\mathbf{N}(\%)$ & Psychotropic drugs & $\mathbf{N}(\%)$ \\
\hline Depression & $\mathbf{4 8}(76.2)$ & Specific antidepressants & $\mathbf{8}(12.7)$ \\
OSAS & $\mathbf{1 7}(27.0)$ & Dopamine agonist & $\mathbf{4}(6.3)$ \\
Diabetes mellitus & $\mathbf{3}(4.8)$ & Typical benzodiazepine & $\mathbf{6}(9.5)$ \\
Alcohol dependence & $\mathbf{3}(4.8)$ & Atypical benzodiazepine & $\mathbf{1 6}(25.4)$ \\
Arterial hypertension & $\mathbf{2 0}(31.7)$ & Neuroleptics & $\mathbf{6}(9.5)$ \\
Coronary heart disease & $\mathbf{4}(6.3)$ & Thyroid medication & $\mathbf{1 1}(17.5)$ \\
Osteoarthritis & $\mathbf{3}(4.8)$ & Antihypertensives & $\mathbf{1 2}(19.0)$ \\
Tricycle antidepressants & $\mathbf{1 9}(30.2)$ & Analgesics & $\mathbf{4}(6.3)$ \\
Serotonin reuptake inhibitors & $\mathbf{8}(12.7)$ & Beta-blockers & $\mathbf{2 ( 3 . 2 )}$ \\
\hline
\end{tabular}

All measures resulting in an accentuation of the day-night-rhythm are indicated in older adults with insomnia. These are primarily day structuring, physical activities in the day, avoidance of long day-sleep, and avoidance of early bedtimes [33].

The most prominent complaints of our patients were difficulties with falling to asleep as well as day time sleepiness. According to the results of the ESS, PSQI and sleep diaries, these two complaints improved significantly, and the time needed to fall asleep was shortened to nearly 30 minutes according to the subjective measures (Sleep diary and PSQI).

However, the analysis of the sleep diaries revealed very low sleep efficiency before as well as after the treatment, whereas sleep efficiency also improved according to the PSQI. 


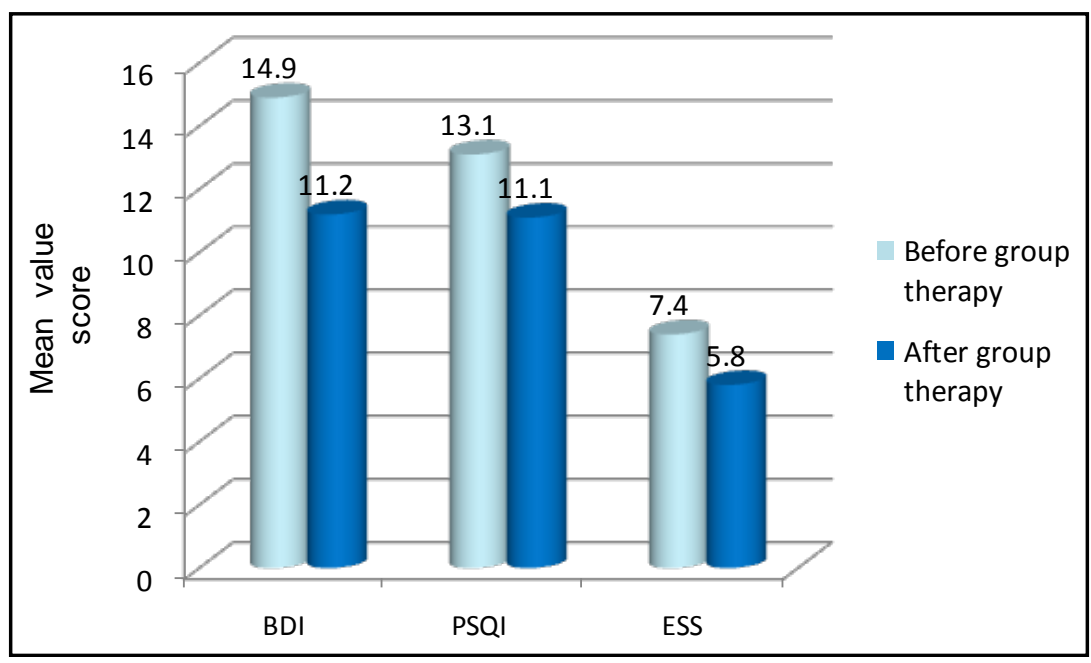

Figure 2. Mean values of the scores of the testing procedures in pre-post-comparison.

The patients spent 8 hours in bed and slept approximately 5.5 hours, which is a very short duration. Therefore, we sought to determine the reason for the improvement of tiredness and sleep quality.

We assume that the subjective improvement of the sleep and daily tiredness can be explained by the fact that 48 (76.2\%) of the patients suffered from light depression at the beginning of the treatment.

After the treatment, the BDI score of 34 patients as well as their WHO-5 score improved and reached an almost non-pathological value, which could explain the positive subjective estimation of sleep (quality) and daily tiredness.

Additional reasons for this subjective improvement include the daily light therapy in the fifth week of the treatment and the social group factors before the end of the treatment.

We conclude that the non-pharmacological combination treatment consisting from CBT, Light Therapy and physical activity can be effective in the Treatment of comorbid insomnia in older adults. In addition, we conclude that a combination of CBT, light therapy and physical activity can also improve the clinical outcome of depression.

We recommend a controlled study trial to verify the effects of the treatment of comorbid insomnia in older adults, using a combination of CBT, bright light, and physical activity with the aim to confirm the efficacy of the combination treatment.

\section{Limitations}

First limitation is that there was no control group due to the naturalistic design of the study. Furthermore, the clinic changed its psychometric standards in connection with general changes in the field, resulting in a switch over from the BDI to the revised BDI II.

Another limitation is the lack of compliance of questionnaire completion. These were used for the psychological assessment, statistical analysis (with an impact because of missing data) and in the implementation of the CBT techniques (modified reduction of the time spent in bed).

\section{Disclosure}

All authors disclosure absence of financial support, any conflict of interest as well as off-label or investigational use.

\section{References}

[1] Bloom, H.G., Ahmed, I., Alessi, C.A., Ancoli-Israel, S., Buysse, D.J., Kryger, M.H., Phillips, B.A., Thorpy, M.J., Vitiello, M.V. and Zee, P.C. (2009) Evidence-Based Recommendations for the Assessment and Management of Sleep Disorders in Older Persons. Journal of the American Geriatrics Society, 57, 761-789.

http://dx.doi.org/10.1111/j.1532-5415.2009.02220.x 
[2] Lichstein, K.L. and Morin, C.M. (2000) Treatment of Late-Life Insomnia. Sage Publications Inc., Thousand Oaks.

[3] Monane, M. (1992) Insomnia in the Elderly. Journal of Clinical Psychiatry, 53, 23-28.

[4] Montgomery, P. and Dennis, J. (2003) Cognitive Behavioural Interventions for Sleep Problems in Adults Aged 60+. Cochrane Database of Systematic Reviews, 1, Article ID: CD003161.

[5] Ring, D. (2001) Management of Chronic Insomnia in the Elderly. Clinical Excellence for Nurse Practitioners, 5, 1316.

[6] Ford, D.E. and Kamerow, D.B. (1989) Epidemiologic Study of Sleep Disturbances and Psychiatric Disorders: An Opportunity for Prevention? The Journal of the American Medical Association, 262, 1479-1484. http://dx.doi.org/10.1001/jama.1989.03430110069030

[7] Morin, C.M. and Benca, R. (2012) Chronic Insomnia. Lancet, 379, 1129-1141. http://dx.doi.org/10.1016/S0140-6736(11)60750-2

[8] Kaneda, R. and Furuta, H. (2009) Insomnia in Old Age. Nippon Rinsho, 67, 1548-1552.

[9] Moller, H.J., Barbera, J., Kaymov, L. and Shapiro, C.M. (2004) Psychiatric Aspects of Late-Life Insomnie. Sleep Medicine Reviews, 8, 31-34. http://dx.doi.org/10.1016/S1087-0792(03)00022-4

[10] Zee, P. and Bloom, H.G. (2006) Understanding and Resolving Insomnia in the Elderly. Geriatrics, 61, 1-12.

[11] Hemmeter, U.M., Thum, A. and Krieg, J.C. (2007) Altersinsomnie. Somnologie, 11, 84-98. http://dx.doi.org/10.1007/s11818-007-0306-8

[12] Wolkove, N., Elkholy, O., Baltzan, M. and Palayew, M. (2007) Sleep and Aging: 1. Sleep Disorders Commonly Found in Older People. Canadian Medical Association Journal, 176, 1299-1304. http://dx.doi.org/10.1503/cmaj.060792

[13] Foley, D.J., Monjan, A.A., Brown, S.L., Simonsick, E.M., Wallace, R.B. and Blazer, D.G. (1995) Sleep Complaints among Elderly Persons: An Epidemiologic Study of Three Communities. Sleep, 18, 425-432.

[14] Ancoli-Israel, S. (2009) Sleep and Its Disorders in Aging Populations. Sleep Medicine, 10, S7-S11. http://dx.doi.org/10.1016/j.sleep.2009.07.004

[15] Graham, D. and McLacham, A. (2004) Declining Melatonin Levels and Older People. How Old Is Old? Neuro Endocrinology Letters, 25, 415-418.

[16] Pandi-Perumal, S.M., Srinivasan, V., Spence, D.W. and Cardinali, D.P. (2007) Role of the Melatonin System in the Control of Sleep: Therapeutic Implications. CNS Drugs, 21, 995-1018. http://dx.doi.org/10.2165/00023210-200721120-00004

[17] Franzen, P.L. and Buysse, D.J. (2008) Sleep Disturbances and Depression: Risk Relationships for Subsequent Depression and Therapeutic Implications. Dialogues in Clinical Neuroscience, 10, 473-481.

[18] Norra, C., Richter, N. and Juckel, G. (2011) Sleep Disturbances and Suicidality: A Common Association to Look for in Clinical Practice and Preventive Care. EPMA Journal, 2, 295-307.

[19] Spiegelhalder, K., Regen, W., Nanovska, S., Baglioni, C. and Riemann, D. (2013) Comorbid Sleep Disorders in Neuropsychiatric Disorders across the Life Cycle. Current Psychiatry Reports, 15, 364. http://dx.doi.org/10.1007/s11920-013-0364-5

[20] Espie, C.A., MacMahon, K.M., Kelly, H.L., Espie, C.A., MacMahon, K.M., Kelly, H.L., Broomfield, N.M., Douglas, N.J., Engleman, H.M., McKinstry, B., Morin, C.M., Walker, A. and Wilson, P. (2007) Randomized Clinical Effectiveness Trial of Nurse-Administrated Small-Group Cognitive Behavioral Therapy for Persistent Insomnia in General Practice. Sleep, 30, 574-584.

[21] Morin, C.M. (1993) Insomnia: Psychological Assessment and Management. Guilford, New York.

[22] Morin, C.M., Colecchi, C., Stone, J., Sood, R. and Brink, D. (1999) Behavioural and Pharmacological Therapies for Late Life Insomnia. JAMA, 281, 991-999. http://dx.doi.org/10.1001/jama.281.11.991

[23] Morin, C.M., Hauri, P.J., Espie, C.A., Spielman, A., Buysse, D.J. and Bootzin, R.R. (1999) Nonpharmacologic Treatment of Chronic Insomnia. An American Academy of Sleep Medicine Review. Sleep, 22, 1134-1156.

[24] Spielman, A.J., Saskin, P. and Thorpy, M.J. (1987) Treatment of Chronic Insomnia by Restriction of Time in Bed. Sleep, 10, 45-56.

[25] Happe, S. and Paulus, W. (2006) Schlafstörungen im Alter. In: Deuschl, G. and Reichmann, H., Eds., Gerontoneurologie, Georg Thieme Verlag, Stuttgart, 85-96.

[26] Joshi, S. (2008) Nonpharmacological Therapy for Insomnia in the Elderly. Clinics in Geriatric Medicine, 24, $107-119$. http://dx.doi.org/10.1016/j.cger.2007.08.005

[27] Fetveit, A. (2009) Late-Life Insomnia: A Review. Geriatrics \& Gerontology International, 9, 220-234. http://dx.doi.org/10.1111/j.1447-0594.2009.00537.x 
[28] Irwin, M.R., Cole, J. and Nicassio, P.M. (2006) Comparative Meta-Analysis of Behavioral Interventions for Insomnia and Their Efficacy in Middle-Aged Adults and in Older Adults 55+ Years of Age. Health Psychology, 25, 3-14. http://dx.doi.org/10.1037/0278-6133.25.1.3

[29] McCurry, S.M., Logsdon, R.G., Teri, L. and Vitiello, M.V. (2007) Evidence-Based Psychological Treatments for Insomnia in Older Adults. Psychology and Aging, 22, 18-27. http://dx.doi.org/10.1037/0882-7974.22.1.18

[30] Riedel, B.W., Lichstein, K.L. and Dwyer, W.D. (1995) Sleep Compression and Sleep Education for Older Insomniacs: Self-Help versus Therapist Guidance. Psychology and Aging, 10, 54-63. http://dx.doi.org/10.1037/0882-7974.10.1.54

[31] Bootzin, R. and Epstein, D. (2000) Stimulus Control. In: Lichstein, K.L. and Morin, C.M., Eds., Treatment of Late-Life Insomnia, Sage Publications Inc, Thousand Oaks.

[32] Morgan, K. (2003) Daytime Activity and Risk Factors for Late-Life Insomnia. Journal of Sleep Research, 12, $231-238$. http://dx.doi.org/10.1046/j.1365-2869.2003.00355.x

[33] Montgomery, P. and Dennis, J. (2002) Physical Exercise for Sleep Problems in Adults Aged 60+. Cochrane Database of Systematic Reviews, 4, Article ID: CD003404.

[34] Mucha, C. (2010) Körperliche Aktivität gegen Schlafstörungen. Klinikarzt, 39, 568-571. http://dx.doi.org/10.1055/s-0031-1271968

[35] Campbell, S.S., Dawson, D. and Anderson, M.W. (1993) Alleviation of Sleep Maintenance Insomnia with Timed Exposure to Bright Light. Journal of the American Geriatrics Society, 41, 829-836.

[36] Lack, L., Wright, H., Kemp, K. and Gibbon, S. (2005) The Treatment of Early-Morning Awakening Insomnia with 2 Evenings of Bright Light. Sleep, 28, 616-623.

[37] Montgomery, P. and Dennis, J. (2002) Bright Light Therapy for Sleep Problems in Adults Aged 60+. Cochrane Database of Systematic Reviews, 2, Article ID: CD003403.

[38] Lichstein, K.L., Wilson, N.M. and Johnson, C.T. (2000) Psychological Treatment of Secondary Insomnia. Psychology and Aging, 15, 232-240. http://dx.doi.org/10.1037/0882-7974.15.2.232

[39] Buysse, D.J. (2004) Insomnia, Depression and Aging. Assessing Sleep and Mood Interactions in Older Adults. Geriatrics, 59, 47-51.

[40] Taylor, D.J., Lichtenstein, K.L., Weinstock, J., Sanford, S. and Temple, J.R. (2007) A Pilot Study of Cognitive-Behavioral Therapy of Insomnia in People with Mild Depression. Behavior Therapy, 38, 49-57. http://dx.doi.org/10.1016/j.beth.2006.04.002

[41] Rybarzyk, B., Stepanski, E., Fogg, K., Lopez, M., Barry, P. and Davis, A. (2005) A Placebo-Controlled Test of CognitiveBehavioral Therapy for Comorbid Insomnia in Older Adults. Journal of Consulting and Clinical Psychology, 73, 11641174. http://dx.doi.org/10.1037/0022-006X.73.6.1164

[42] Jakobson, E. (1929) Progressive Relaxation. University of Chicago Press, Chicago.

[43] Buysse, D.J., Reynolds, C.F., Monk, T.H., Berman, S.R. and Kupfer, D.J. (1989) The Pittsburgh Sleep Quality Index (PSQI): A New Instrument for Psychiatric Practice and Research. Psychiatry Research, 28, 193-213. http://dx.doi.org/10.1016/0165-1781(89)90047-4

[44] Johns, M.W. (1991) A New Method for Measuring Daytime Sleepiness: The Epworth Sleepiness Scale. Sleep, 14, 540545.

[45] Hautzinger, M., Bailer, M., Worall, H. and Keller, F. (1995) BDI Beck-Depressions-Inventar. Testhandbuch 2, überarbeitete Auflage. Verlag Hans Huber, Bern.

[46] Hautzinger, M., Keller, F. and Kühner, C. (2009) BDI-II. Beck-Depressions-Inventar. Revision. 2. Auflage. Pearson Assessment, Frankfurt.

[47] Heun, R., Burkart, M., Maier, W. and Bech, P. (1999) Internal and External Validity of the WHO Well-Being Scale in the Elderly General Population. Acta Psychiatrica Scandinavica, 99, 171-178. http://dx.doi.org/10.1111/j.1600-0447.1999.tb00973.x 


\section{Note List of Abbreviations}

CBT: cognitive behavioral therapy EPESE: established population of epidemiologic studies of the elderly BD: beck depressions inventory SPSS

ESS: epworth sleepiness scale

PSQI: pittsburgh sleep quality index

WHO: 5-questionnaire on general well-being 